\title{
Explaining cosmic ray antimatter with secondaries from old supernova remnants
}

\author{
Philipp Mertsch, ${ }^{a, *}$ Andrea Vittino $^{a}$ and Subir Sarkar ${ }^{b}$ \\ ${ }^{a}$ Institute for Theoretical Particle Physics and Cosmology (TTK), \\ RWTH Aachen University, 52056 Aachen, Germany \\ ${ }^{b}$ Rudolf Peierls Centre for Theoretical Physics, University of Oxford, \\ Parks Road, Oxford OX1 3PU, UK \\ E-mail: pmertsch@physik.rwth-aachen.de
}

\begin{abstract}
Despite significant efforts over the last decade, the origin of the cosmic ray positron excess has still not been unambiguously established. A popular class of candidates are pulsars or pulsar wind nebulae but these cannot account for the observed hard spectrum of cosmic ray antiprotons. We revisit the alternative possibility that the observed high-energy positrons are secondaries created by spallation in supernova remnants during the diffusive shock acceleration of the primary cosmic rays, which are further accelerated by the same shocks. The resulting source spectrum of positrons at high energies is then naturally harder than that of the primaries, as is the spectrum of other secondaries such as antiprotons. We present the first comprehensive investigation of the full parameter space of this model - both the source parameters as well as those governing galactic transport. Various parameterisations of the cross-sections for the production of positrons and antiprotons are considered, and the uncertainty in the model parameters discussed. We obtain an excellent fit to the recent precision measurements by AMS-02 of cosmic ray protons, helium, positrons and antiprotons, as well as of various primary and secondary nuclei. The only notable deviation is an excess of antiprotons around $10 \mathrm{GeV}$. This model thus provides an economical explanation of the spectra of all secondary species - from a single well-motivated population of sources.
\end{abstract}

$3^{\text {th }}$ International Cosmic Ray Conference (ICRC 2021)

July 12 th - 23rd, 2021

Online - Berlin, Germany

\footnotetext{
${ }^{*}$ Presenter
} 


\section{Introduction}

One of the pillars of our current understanding of Galactic cosmic rays (CRs) [1] is their diffusive transport through the interstellar medium (ISM). Closely related is the notion that certain speciea, called secondaries, that are overabundant in CRs compared to solar abundances, are created through inelastic collisions of (mostly) heavier CR species, called primaries, with the gas in the ISM. Prototypical examples for secondaries are boron, beryllium and lithium that are produced from predominantly primary carbon and oxygen. The central parameter in the production of secondaries is the grammage $\lambda$, that is the average amount of matter traversed by CRs, which is proportional to the residence time of CRs $\tau$. The grammage can be estimated from the measured ratios of secondaries and primaries, like the boron-to-carbon (B/C) ratio. In a model with homogeneous and isotropic diffusion, the residence time and hence the grammage will be inversely proportional to the diffusion coefficient. Due to the typical power law dependence of the diffusion coefficient on rigidity $\mathcal{R}$, e.g. $\kappa \propto \mathcal{R}^{\delta}$ in the relativistic regime, the grammage will decrease in a similar fashion, i.e. $\lambda \propto \mathcal{R}^{-\delta}$. Broadly speaking, this trend is observed in the measured nuclear secondary-to-primary ratios above $\sim 10 \mathrm{GV}$ even though other effects are important at lower rigidities.

What is ignored in this picture is the possibility that a part of the measured grammage is not accumulated in the ISM, but in or close to the sources of CRs, e.g. supernova remnants (SNRs). This is commonly justified by the fact that the residence time inside the sources, which is bounded from above by the maximum age of sources, typically $t_{\mathrm{age}} \sim O\left(10^{4}\right) \mathrm{yr}$ for SNRs, is much shorter than the residence time in the Galaxy, $\tau$ which is typically $\tau \sim O\left(10^{7}\right)$ yr at GV rigidities, as measured from a combination of stable and unstable nuclear ratios. Even allowing for the possibility of a gas density that is enhanced with respect to the average Galactic value, maybe by a factor of 10 , the grammage from the source seems negligible.

At close inspection, however, this argument appears to be flawed, mainly due to the fact that the energy dependence of the source grammage will likely be different from that of the ISM grammage. One reason for this is the typical embedding of SNRs in regions of enhanced gas density, e.g. in molecular cloud complexes. Upon leaving those regions, CR particles would have already accumulated a grammage proportional to their residence time and the average ambient gas density in the cloud. Note however, that the flux of secondary CRs from the cloud would not be softer than the primary flux and so this would contribute with a rigidity-independent contribution to the total grammage. With the galactic contribution falling as a power law in rigidity, the relative importance of the near-source-produced secondaries at higher rigidities would be much larger than the ratio of abundances at $\mathrm{GV}$ rigidities implies. Another reason for an additional contribution to the grammage from nearby the source would be in models with a reduced diffusivity in the near source region, for instance due to turbulence self-generated by the CRs. (See, however, Ref. [2].)

More dramatically still would be the underestimate of the source abundance if the secondaries were able to participate in the shock acceleration of the CR source. It can be shown that in the simplest test particle picture of diffusive shock acceleration, the secondaries will acquire a harder spectrum then their primaries [3-8]. This is particularly pronounced for old SNRs with low shock speeds. Expressed in the language of grammages, this corresponds to a grammage that is not falling, but rising with rigidity. It is easy to imagine that the total grammage in particular at hundreds of GV will be naturally much enhanced above the previous estimates. 
The latter idea has gained some currencies in the wake of various anomalies in CR secondaries, like positrons [9, 10] and antiprotons [11]. While these excesses have generated a lot of attention for indirect detection of particle dark matter, also pulsars have been suggested as an astrophysical source, but these cannot account for the unexpectedly hard antiproton spectrum [12]. Old SNRs can readily explain excesses in both positrons and antiprotons and in fact all secondary species without the introduction of a new class of sources. This makes this model economical and predictive. However, the propagation model parameters, which usually get calibrated by fitting of (secondary) nuclei, cannot be kept fixed, unlike in the study of dark matter or pulsar sources. Given the absence of a model of CRs that can consistently explain secondary species like positrons, antiprotons and nuclear secondaries like boron, we therefore revisited this class of models and performed a systematic parameter study.

\section{Model}

\subsection{Acceleration of cosmic ray secondaries in the sources}

We treat the production of secondaries from and their acceleration alongside primary CRs in the testparticle approxiation of DSA. For a non-relativistic, planar shock, the phase-space density $f_{i}$ of a CR species $i$ can be described by the CR transport equation, formulated in the shock frame,

$$
\frac{\partial f_{i}}{\partial t}=-u \frac{\partial f_{i}}{\partial x}+\frac{\partial}{\partial x} D_{i} \frac{\partial f_{i}}{\partial x}-\frac{p}{3} \frac{d u}{d x} \frac{\partial f_{i}}{\partial p}-\Gamma_{i} f_{i}+q_{i} .
$$

Here, $u$ denotes the advection velocity which is constant on either side of the shock, $D_{i}$ is the spatially constant diffusion coefficient, which we parametrised as

$$
D=3 \times 10^{22} \mathrm{~cm}^{2} \mathrm{~s}^{-1} \beta K_{\mathrm{B}}\left(\frac{B}{1 \mu \mathrm{G}}\right)^{-1}\left(\frac{\mathcal{R}}{1 \mathrm{GV}}\right)^{\alpha} .
$$

$\Gamma_{i}$ is the rate of destruction of species $i$ by spallation and $q_{i}$ denotes a source term, either due to injection (for primaries only) or from spallation (for secondaries). The shock is assumed to be at $x=0$ and in the following we denote quantities on the upstream $(x<0)$ and downstream $(x>0)$ sides with "+" and "-", respectively. Note that the gas densities $n_{ \pm}$and velocities $u_{ \pm}$on either side of the shock are related by $r=n_{+} / n_{-}=u_{-} / u_{+}$, where $r$ denotes the compression ratio. As has been shown before (e.g. $[5,6,8]$ ), the steady-state phase-space density on the downstream side is not spatially constant, but contains a term linear in the distance from the shock,

$$
f_{i}^{+}(x, p)=f_{i}^{0}(p)+r\left(q_{i}^{0}(p)-\Gamma_{i}^{-} f_{i}^{0}(p)\right) \frac{x}{u_{+}} .
$$

The injection term and the phase space density at the shock are called $q_{i}^{0}(p)$ and $f_{i}^{0}(p)$, respectively, and one finds

$$
f_{i}^{0}(p)=\int_{0}^{p} \frac{d p^{\prime}}{p^{\prime}}\left(\frac{p^{\prime}}{p}\right)^{\gamma} e^{-\gamma\left(1+r^{2}\right)\left(D_{i}(p)-D_{i}\left(p^{\prime}\right)\right) \frac{\Gamma_{i}(p)}{u_{-}^{2}}}\left[\gamma\left(1+r^{2}\right) \frac{D_{i}\left(p^{\prime}\right)}{u_{-}^{2}} q_{i}^{0}\left(p^{\prime}\right)+\gamma Y_{i} \delta\left(p^{\prime}-p_{0}\right)\right] .
$$

In the derivation of these results, we assumed that $\Gamma_{i} D_{i} / u_{ \pm}^{2} \ll 1$ and $x \Gamma_{i} / u_{ \pm}<x_{\max } \Gamma_{i} / u_{ \pm} \ll 1$.

The particle spectrum $\mathrm{d} N / \mathrm{d} p$ that is released into the ISM at the end of the SNR's life is the integral of $4 \pi p^{2} f$ over the downstream volume $V=4 / 3 \pi\left(u_{+} \tau_{\mathrm{SNR}}\right)^{3}$ of the shock:

$$
\frac{d N_{i}}{d p}=4 \pi \int_{0}^{\tau_{\mathrm{SNR}} u_{+}} d x x^{2} 4 \pi p^{2} f_{i}(x, p)=4 \pi V^{2}\left[f_{i}^{0}+\frac{3}{4} r \tau_{\mathrm{SNR}}\left(q_{i}^{0}-\Gamma_{i}^{-} f_{i}^{0}\right)\right] .
$$




\subsection{Cosmic-ray propagation and solar modulation}

The transport of CRs in the ISM can be described by a transport equation similar to eq. (1), but using eq. (5) as a source term. The transport parameters, in particular the diffusion coefficient and the gas densities have to be adapted to the conditions of the ISM. In addition, the effect of secondorder Fermi acceleration due to momentum diffusion, commonly know as diffusive reacceleration, needs to be considered. We have adopted the GALPROP code [13] and adapted it in several respects.

In order to accommodate breaks observed in primary and secondary spectra around $300 \mathrm{GV}$, we have allowed for spectral breaks in the diffusion coefficient,

$$
D_{x x}(\mathcal{R})=D_{0} \beta\left(\frac{\mathcal{R}}{\mathcal{R}_{1}}\right)^{\delta_{1}} \prod_{i=1}^{2}\left(1+\left(\frac{\mathcal{R}}{\mathcal{R}_{i}}\right)^{1 / s_{i}}\right)^{s_{i}\left(\delta_{i+1}-\delta_{i}\right)} .
$$

While the high-rigidity break could be explained by a transition in the turbulence spectrum responsible for CR diffusion [14], the break at lower rigidities could be due to damping of said turbulence [15] if the distribution at the relevant rigidities was more isotropic. In addition, we have extended the range of production cross-sections available in GALPROP by a number of recent parametrisations. We refer the interested reader to Ref. [16] for a more detailed presentation.

Finally, we have modelled solar modulation in the force-field approximation [17], but allowing for different Fisk potentials for different CR species. The flux for each combination of parameters is therefore the result of first a computationally expensive GALPROP run followed by second a computationally inexpensive force-field modulation and overall normalisation of all spectra.

\subsection{Model parameters and data}

Our model, as presented in Secs. 2.1 and 2.2, comprises 23 parameters in total, out of which 12 relate to the source, 7 to the propagation in the ISM and 4 to solar modulation. We list these parameters together with their best-fit values in Tbl. 1. Note that the low-energy source spectral indices of helium and heavier nuclei have been fixed to $\left(\gamma_{1}^{p}-0.08\right)$ where $\gamma_{1}^{p}$ is the proton lowenergy spectral index. We have also related the break rigidities of heavier species to the proton one, $\mathcal{R}_{\mathrm{br}}^{p}$, assuming these breaks to emulate the effect of source discretenes [18]. The best-fit values have been determined by minimising the total $\chi^{2}$ with respect to data from Voyager 1 and AMS-02. Given possible correlations between parameters and due to the existence of local minima in the high-dimensional parameter space, we have adopted a Monte Carlo Markov Chain algorithm [19] for sampling of the posterior distribution.

\section{Results}

In Fig. 1, we present our best-fit fluxes of CR protons, helium, positrons, antiprotons, carbon, oxygen, boron and nitrogen as well as the boron-to-carbon ratio. For proton, helium, carbon and boron in particular, we show both the data from Voyager 1 [20] (as a function of kinetic energy per nucleon) and the AMS-02 data [21] (as a function of rigidity) in separate panels. For the remaining species, that is positrons, antiprotons, oxygen and nitrogen, we compare to the AMS-02 data $[11,22]$ only. In each panel, we have indicated the reduced $\chi^{2}$-value for this observable alone 
Table 1: Free parameters and their best-fit values.

\begin{tabular}{|c|c|c|}
\hline \multicolumn{3}{|r|}{ Source parameters } \\
\hline$\tau_{\mathrm{SNR}}$ & $=\left(2.569_{-0.300}^{+0.318}\right) \times 10^{4}$ & source age in yr \\
\hline$K_{\mathrm{B}}$ & $=\left(5.221_{-0.597}^{0.797}\right) \times 10^{2}$ & normalisation of source diffusion coefficient, see eq. (2) \\
\hline $\mathcal{R}_{\max }$ & $=\left(9.838_{-1.268}^{+1.405}\right) \times 10^{2}$ & maximum rigidity in $\mathrm{GV}$ \\
\hline$\alpha$ & $=0.699_{-0.016}^{+0.012}$ & spectral index of diffusion coefficient \\
\hline$\gamma_{1}^{p}$ & $=1.555_{-0.078}^{+0.071}$ & proton spectral indices below $\mathcal{R}_{\mathrm{br}}^{p}$ \\
\hline$\gamma_{2}^{p}$ & $=2.303_{-0.006}^{+0.018}$ & proton spectral indices above $\mathcal{R}_{\mathrm{br}}^{p \mathrm{pr}}$ \\
\hline $\log _{10}\left[\mathcal{R}_{\mathrm{br}}^{p} / \mathrm{GV}\right]$ & $=2.997_{-0.069}^{+0.060}$ & break rigidity \\
\hline$s_{12}$ & $=2.053_{-0.156}^{+0.009}$ & softness of break at $\mathcal{R}_{\mathrm{br}}^{p}$ \\
\hline$\gamma_{2}^{\mathrm{He}}$ & $=2.246_{-0.004}^{+0.004}$ & Helium spectral index above $\mathcal{R}_{\mathrm{br}}^{\mathrm{He}}$ \\
\hline$\gamma_{2}^{\mathrm{C}}$ & $=2.252_{-0.005}^{+0.005}$ & Carbon spectral index above $\mathcal{R}_{\mathrm{br}}^{\mathrm{Cr}}$ \\
\hline$\gamma_{2}^{\text {nuc }}$ & $=2.284_{-0.004}^{+0.004}$ & Nuclear spectral index above $\mathcal{R}_{\mathrm{br}}^{\text {nuc }}$ \\
\hline$N$ & $=0.994_{-0.001}^{+0.001}$ & Overall scaling factor \\
\hline \multicolumn{3}{|r|}{ Galactic parameters } \\
\hline$D_{0}$ & $=3.487_{-0.088}^{+0.102}$ & normalisation of diffusion coefficient in $10^{28} \mathrm{~cm}^{2} \mathrm{~s}^{-1}$ \\
\hline$\delta_{1}$ & $=-0.238_{-0.036}^{+0.034}$ & spectral index of diffusion coefficient below $\mathcal{R}_{12}$ \\
\hline $\log _{10}\left[\mathcal{R}_{12} / \mathrm{GV}\right]$ & $=3.684_{-0.013}^{+0.012}$ & first break rigidity \\
\hline$s_{12}^{\text {diff }}$ & $=0.196_{-0.022}^{+0.026}$ & softness of break at $\mathcal{R}_{12}$ \\
\hline$\delta_{2}$ & $=0.554_{-0.005}^{+0.005}$ & spectral index of diffusion coefficient between $\mathcal{R}_{12}$ and $\mathcal{R}_{23}$ \\
\hline$s_{23}^{\text {diff }}$ & $=0.098_{-0.012}^{+0.014}$ & softness of break at $\mathcal{R}_{23}$ \\
\hline$v_{A}$ & $=9.707_{-1.398}^{+2.099}$ & Alfvén speed in $\mathrm{km} \mathrm{s}^{-1}$ \\
\hline \multicolumn{3}{|c|}{ Solar modulation parameters } \\
\hline$\phi_{p}$ & $=0.595_{-0.003}^{+0.003}$ & Fisk potential for protons in GV \\
\hline$\phi_{e^{+}}$ & $=0.471_{-0.002}^{+0.002}$ & Fisk potential for positrons in $\mathrm{GV}$ \\
\hline$\phi_{\bar{p}}$ & $=0.588_{-0.018}^{+0.018}$ & Fisk potential for antiproton in GV \\
\hline$\phi_{\text {nuc }}$ & $=0.608_{-0.003}^{+0.003}$ & Fisk potential for nuclei in GV \\
\hline
\end{tabular}

(not attributing any parameters to any observable in particular). For the overall $\chi^{2}$, we have found a value of 478.5 for 589 data points, resulting in a reduced $\chi_{\mathrm{r}}^{2}=478.5 /(589-24) \simeq 0.85$.

The $\chi_{\mathrm{r}}^{2}$-values for individual data sets are generally excellent as well. We note that the values found for the proton and helium fluxes measured by AMS ( 0.5 and 0.3 , respectively) are very small. For the computation of $\chi^{2}$ 's we have added the statistical and systematic errors in quadrature, which can lead to an overestimate if the systematic errors are correlated. The $\chi_{\mathrm{r}}^{2}$ for carbon and oxygen are equally small ( 0.6 and 0.3 , respectively), most likely for the same reason.

As for the fits of primary species to Voyager 1 data, the fit is excellent for protons, which too raises questions about the possibility of unaccounted for correlations. The fit to heavier nuclei are generally less satisfactory. We remind the reader that our treatment of the low-energy break in the source spectrum is meant to emulate the effect of source stochasticity on the predicted spectra. Our heuristic scaling of the rigidity of the break for different species is allowing for the general trend in the measured fluxes, i.e. a peak between 10 and $100 \mathrm{MeV} / \mathrm{n}$, to be reproduced, but including this effect properly into our analysis could possibly improve the quality of the fit.

As for secondary nuclei, we find an acceptable fit to the boron data as measured by Voyager 1. 

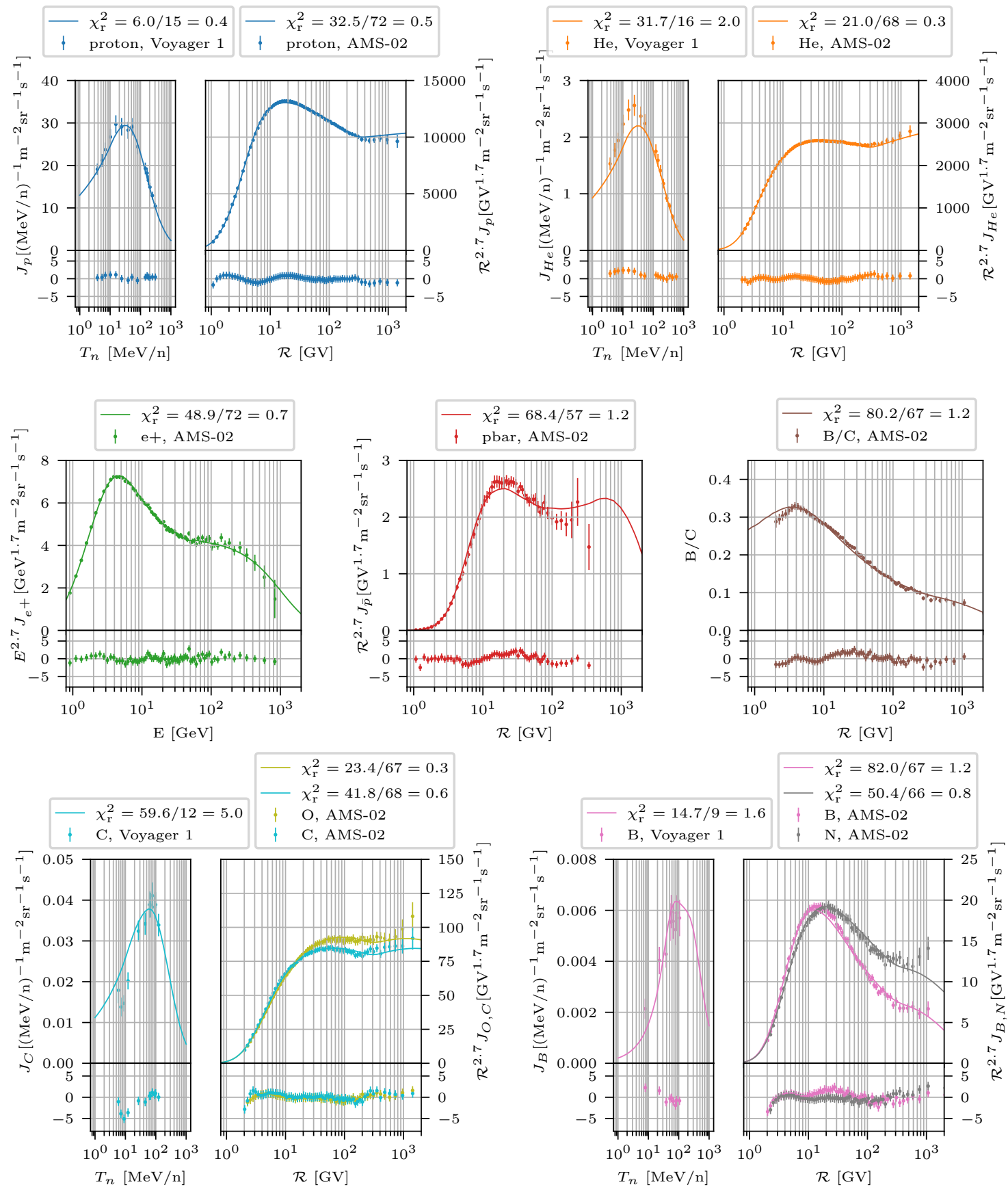

Figure 1: Best-fit spectra for various CR primaries and secondaries: protons (top left), helium (top right), positrons (middle left), antiprotons (middle centre), boron-to-carbon ratio (middle right), carbon and oxygen (bottom left), boron and nitrogen (bottom right).

As for the AMS-02 data, while the $\chi^{2}$ is again very good, an excess around a few tens of GV is notable. (This is also visible in the boron-to-carbon ratio.) We note that for nuclear species, we have not accounted for any uncertainties in the nuclear cross-sections and warn that deviations from the fits and parametrisations employed in GALPROP could plausibly explain deviations of this size. 


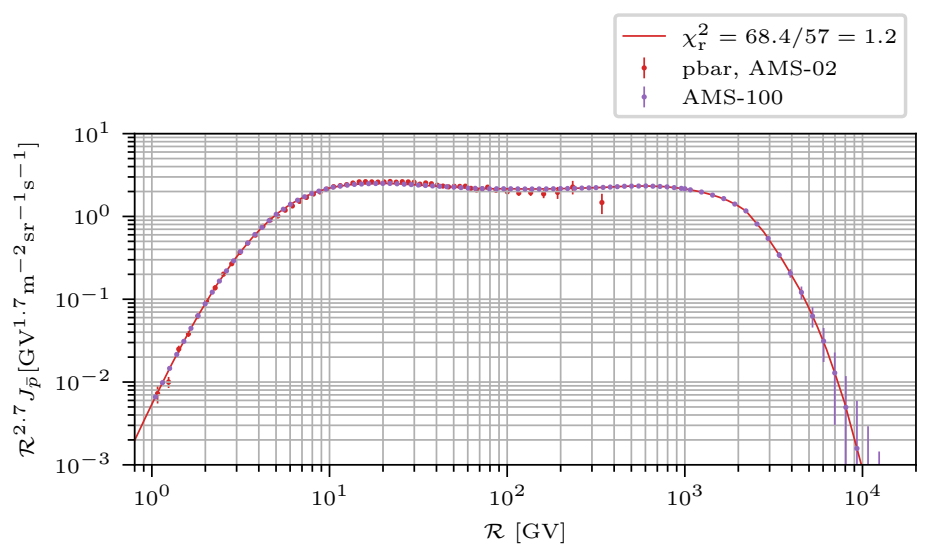

Figure 2: Antiproton spectrum of Fig. 1, together with the data expected for the proposed AMS-100 experiment [27].

The reproduction of the positron data measured by AMS-02 finally is again very good $\left(\chi_{\mathrm{r}}^{2} \simeq\right.$ 0.7). The fit to the anti-proton flux is markedly improved over earlier studies that did not explore the full parameter space (e.g. [8, 23]). An excess around a few tens of GV is striking though. We note in passing that this might be the same excess that has been noted in models of Galactic CRs without any secondaries produced and accelerated inside sources [24]. We caution, however, that there have been indications that this feature might be exaggerated due to correlated errors, too [25, 26].

\section{Summary and conclusions}

We have presented the results of a systematic parameter study of the acceleration of secondaries in old SNRs. In doing so, we have improved over previous studies by extending the data set considered to include the latest AMS-02 data on protons, helium, carbon, oxygen, boron, nitrogen, positrons and antiprotons and Voyager 1 data on protons, helium, boron and carbon. We have also updated the cross-section parametrisations included in the GALPROP code. The model that we present comprises the best-fit parameters to all those data and provides an excellent fit. In particular, we reproduce the flux of positrons and antiprotons as measured by AMS-02.

We conclude by considering the probably most striking feature of our model, a rather broad bump in the flux of antiprotons around a few hundred GV. While we caution that this feature is very sensitive to the cross-section adopted, we nevertheless might ask what it would take to confirm it observationally in order to further investigate the positron excess. The ideal instrument for this study would be the proposed AMS-100 experiment [27]. In Fig. 2, we show our best-fit antiproton flux together with simulated data for AMS-100, clearly showing how this experiment could confirm or falsify our model, thereby unambiguously clarifying the origin of the positron excess.

\section{References}

[1] S. Gabici et al., IJMP D 28 (2019) no.15, 1930022 [arXiv:1903.11584 [astro-ph.HE]].

[2] S. Recchia et al., [arXiv:2106.04948 [astro-ph.HE]]. 
[3] E. G. Berezhko et al., A\&A 410 (2003), 189-198 [arXiv:astro-ph/0308199 [astro-ph]].

[4] P. Blasi, PRL 103 (2009), 051104 [arXiv:0903.2794 [astro-ph.HE]].

[5] P. Mertsch and S. Sarkar, PRL 103 (2009), 081104 [arXiv:0905.3152 [astro-ph.HE]].

[6] M. Ahlers, P. Mertsch and S. Sarkar, PRD 80 (2009), 123017 [arXiv:0909.4060 [astro-ph.HE]].

[7] N. Tomassetti and F. Donato, A\&A 544 (2012), A16 [arXiv:1203.6094 [astro-ph.HE]].

[8] P. Mertsch and S. Sarkar, PRD 90 (2014), 061301 [arXiv:1402.0855 [astro-ph.HE]].

[9] O. Adriani et al. [PAMELA], Nature 458 (2009), 607-609 [arXiv:0810.4995 [astro-ph]].

[10] M. Aguilar et al. [AMS], PRL 110 (2013), 141102 M. Aguilar et al. [AMS], PRL 122 (2019) no. 4,041102

[11] M. Aguilar et al. [AMS], PRL 117 (2016) no.9, 091103

[12] P. Lipari, PRD 99 (2019) no.4, 043005 [arXiv:1810.03195 [astro-ph.HE]].

[13] I. V. Moskalenko and A. W. Strong, ApJ 493 (1998), 694[arXiv:astro-ph/9710124 [astro-ph]].

[14] P. Blasi et al., PRL 109 (2012), 061101 [arXiv:1207.3706 [astro-ph.HE]].

[15] V. S. Ptuskin et al., ApJ 642 (2006), 902-916 [arXiv:astro-ph/0510335 [astro-ph]].

[16] P. Mertsch, A. Vittino and S. Sarkar, [arXiv:2012.12853 [astro-ph.HE]].

[17] L. J. Gleeson and W. I. Axford, ApJ 154 (1968), 1011

[18] V. H. M. Phan et al., [arXiv:2105.00311 [astro-ph.HE]].

[19] D. Foreman-Mackey et al., Publ. Astron. Soc. Pac. 125 (2013), 306-312 [arXiv:1202.3665 [astro-ph.IM]].

[20] A. C. Cummings et al., ApJ 831 (2016) no.1, 18

[21] M. Aguilar et al. [AMS], PRL 114 (2015), 171103; PRL 119 (2017) no.25, 251101; PRL 120 (2018) no.2, 021101

[22] M. Aguilar et al. [AMS], PRL 113 (2014), 121102

[23] I. Cholis and D. Hooper, PRD 89 (2014) no.4, 043013 [arXiv:1312.2952 [astro-ph.HE]].

[24] A. Cuoco et al., PRL 118 (2017) no.19, 191102 [arXiv:1610.03071 [astro-ph.HE]].

[25] M. Boudaud et al., Phys. Rev. Res. 2 (2020) no.2, 023022 [arXiv:1906.07119 [astro-ph.HE]].

[26] J. Heisig et al., Phys. Rev. Res. 2 (2020) no.4, 043017 [arXiv:2005.04237 [astro-ph.HE]].

[27] S. Schael et al., Nucl. Instrum. Meth. A 944 (2019), 162561 doi:10.1016/j.nima.2019.162561 [arXiv:1907.04168 [astro-ph.IM]]. 